\title{
Intimidad y confidencialidad en el ámbito sanitario. Una perspectiva ética
}

\author{
Privacy and confidentiality in the health field. An ethical perspective
}

Constantino González Quintana*

\section{RESUMEN}

La intimidad y la confidencialidad son dos dimensiones básicas que permiten a cada cual ser uno mismo, comunicarse y relacionarse con los demás. En el ámbito sanitario adquieren un significado especial a causa de la naturaleza de la relación clínica, del número de profesionales que de un modo u otro participan en el acto clínico, y del uso cotidiano de datos personales de salud que gozan de un alto grado de protección. Las páginas siguientes intentan ofrecer una lectura de esos temas desde la perspectiva ética, poniendo el acento en la intimidad, la confidencialidad y el secreto profesional así como en la necesidad de mantener despierto el sentido crítico sobre los riesgos de deshumanización que pueden darse al respecto en la actividad diaria de los profesionales sanitarios. La intimidad y la confidencialidad son dos requisitos para llevar a cabo una asistencia sanitaria de calidad humana y científica.

Palabras clave: Intimidad, confidencialidad, datos de salud, secreto profesional, confianza, bioética.

\begin{abstract}
Privacy and confidentiality are two basic dimensions that allow each one to be oneself and also communicate and interact with others. In the health field they acquire a special meaning because of the very nature of the clinical relationship, the number of professionals who in one way or another participate in the clinical Act, and the daily use of personal health data that enjoy a high degree of protection. The following pages want to offer a reading of these topics from an ethical perspective, emphasizing on the moral privacy, confidentiality and professional secrecy aspect, as well as the need to keep awake the critical sense of the risks of dehumanization that can be given in the daily activity of the professionals. Privacy and confidentiality are two requirements to carry out health care a human and scientific quality healthcare.
\end{abstract}

Keywords: Privacy, confidentiality, health data, professional secret, trust, bioethics.
* Doctor en Filosofía. Profesor de Ética y Filosofía. Titular del Blog Académico: Bioética desde Asturias. Oviedo. Asturias. España.

Correspondencia: CGQ, constantinoq147@ gmail.com https://www. bioeticadesdeasturias. $\mathrm{com} /$

\section{Conflicto de intereses:}

El autor declara que no tiene.

Citar como: González

QC. Intimidad y

confidencialidad en el ámbito sanitario. Una perspectiva ética. Rev CONAMED. 2020; 25(3):

123-128. https://dx.doi. org/10.35366/95984

Financiamiento: Ninguno.

Recibido: 11/08/2020. Aceptado: 08/09/2020.

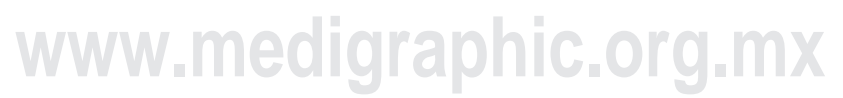




\section{INTRODUCCIÓN}

La existencia de un espacio interior e íntimo de las personas y la información que configura su vida privada gozan en la actualidad del más alto nivel de protección ante cualquier injerencia externa o frente a la difusión no autorizada de la información personal correspondiente. La intimidad y la confidencialidad son dos requisitos para llevar a cabo una asistencia sanitaria de calidad humana y científica.

\section{ALGUNOS CONCEPTOS BÁSICOS}

Intimidad es la cualidad de «íntimo» (latín: intimus) y se refiere a lo más interior o interno donde se desarrollan las facetas singularmente reservadas de la vida de una persona, de un grupo o de una familia.

La privacidad es un concepto específicamente anglosajón (privacy), pero al menos en gran parte coincide con lo que se entiende en lengua española por intimidad, dado que a partir de su origen latino (privatus) se refiere al ámbito de la vida privada al que se tiene derecho a proteger de cualquier intromisión.

La confidencialidad es la cualidad de «confidencial», referida a lo que se hace o se dice en la confianza de que se mantendrá reservado. Proviene del latín (confidentia) y significa revelación secreta, noticia reservada y confianza estrecha e íntima.

El secreto (del latín: secretum) se refiere a lo que se tiene cuidadosamente reservado y oculto, adquiere rasgos específicos cuando es «profesional»: deber que tienen los miembros de ciertas profesiones de no revelar los hechos ni la información que han conocido en el ejercicio de su profesión. En el ámbito sanitario se remonta a Hipócrates.

Datos relativos a la salud son «datos personales relativos a la salud física o mental de una persona física, incluida la prestación de servicios de atención sanitaria que revelen información sobre su estado de salud».2

\section{FORMAS O DIMENSIONES DE LA INTIMIDAD}

De la información: protege lo más interior y reservado de la persona frente a intromisiones ajenas; se refiere a la vida individual, a su entorno personal y familiar así como a la información sobre esos aspectos de la vida personal.

Confidencial: forma específica de la intimidad de la información, que consiste en no divulgar información o datos de la intimidad personal obtenidos confidencialmente.

Física o espacial: se refiere al espacio físico donde se desarrolla la vida íntima con sus ideas, valores, experiencias y sentimientos.

Corporal: forma específica de la intimidad física, consiste en no ser observados ni tocados sin consentimiento. Se refiere tanto al cuerpo como al recato y al pudor de cada uno según su escala de valores. Tiene especial importancia en el ámbito sanitario.

El acceso no autorizado al espacio privado de la vida de una persona y a su correspondiente información vulnera la intimidad, y el hecho de divulgar sin permiso datos personales referentes a la intimidad vulneran la confidencialidad.

\section{ÉTICA DE LA INTIMIDAD Y LA CONFIDENCIALIDAD}

La intimidad y la comunicación son dos ejes básicos de las relaciones humanas. Hay que interpretarlas desde la intersubjetividad porque están presentes de un modo u otro en toda relación interpersonal. Desde una visión general tienen una doble estructura:

- Ámbito interpersonal: se refiere a un círculo íntimo de personas que goza de una atmósfera especial, sin extraños, que están y se sienten juntos. La intimidad hay que situarla sobre todo en el plano del «ser» personas íntimas.

- Ámbito personal: implica proteger ante extraños lo más interior y reservado de la propia vida que, interpretada desde la intersubjetividad, nos sitúa ante una relación entre personas «íntimas» que se debe guardar confidencialmente.

Ahora bien, la intimidad y la comunicación son también dos ejes de la relación clínica basada, principalmente, en la confianza, es decir, en la seguridad recíproca de guardar reserva sobre lo que se hace o se dice en esa relación específica. Visto entonces desde la perspectiva sanitaria tiene también dos ámbitos: 
- Ámbito interpersonal: se refiere a la relación enfermo-profesional sanitario, única en el mundo, cuya atmósfera especial está basada en la confianza con fines exclusivamente sanitarios. La lealtad y la fidelidad son otros valores importantes.

- Ámbito personal: se refiere a la necesidad de revelar información íntima que, interpretada desde la intersubjetividad enfermo-profesional, da lugar a una relación entre sujetos «confidentes» que confían datos personales de salud con la seguridad de que se guardarán confidencialmente.

En resumen, el valor ético de la intimidad reposa en lo más interior y reservado de cada uno, en los valores identitarios que modulan el grado de relación y convivencia. La confidencialidad protege ese valor ético ante intromisiones ajenas. Por su parte, en el ámbito sanitario, donde se desvelan aspectos singularmente reservados de la vida privada, el valor ético de la intimidad está protegido por la obligación de salvaguardar las confidencias de la relación clínica, es decir, por la regla moral de la confidencialidad profesional e institucional. A este propósito viene bien recordar que «no hay medicina sin confianza, ni confianza sin confidencias, ni confidencias sin secreto». ${ }^{3}$

\section{INTIMIDAD CORPORAL EN EL ÁMBITO SANITARIO}

La exposición corporal del enfermo, la observación y el contacto físico es un medio ordinario de la actividad sanitaria para responder a ese «modo anómalo y aflictivo del vivir personal» que es la enfermedad, como decía Pedro Laín Entralgo. ${ }^{4}$ El respeto a la intimidad corporal presenta aquí varias exigencias morales:

- No ir más allá de lo estrictamente imprescindible, incurriendo en exposiciones o contactos físicos innecesarios.

- Solicitar permiso para realizar cualquier exploración, aunque sea un supuesto ordinario del proceso asistencial.

- Llamar antes de entrar en cualquier zona de consultas profesional-enfermo.

- Actualizar constantemente medidas para proteger la intimidad corporal.
- Mostrar especial cuidado cuando intervienen profesionales sanitarios, no sanitariosy alumnos que «deberán respetar la intimidad y dignidad de los pacientes, adoptando actitudes que se ajusten a las normas de cortesía y amabilidad generalmente aceptadas y utilizando un lenguaje adecuado». ${ }^{5}$

Hay que dejar constancia, sin embargo, de que el contacto físico es «lenguaje». El rostro, los sentidos y el cuerpo hablan, expresan múltiples sentimientos y emiten multitud de señales de lenguaje no verbal. Por tanto, no deberíamos vulgarizar ni reducir el cuerpo humano a un mero tecnicismo clínico. Estar atentos a que ese tipo de lenguaje implica todo un modo de entender y practicar la ética de las profesiones sanitarias.

\section{CONFIDENCIALIDAD EN EL ÁMBITO SANITARIO}

La confianza vivida en la relación clínica es la base de donde surge la acción de recoger información personal referente a la salud. Luego vendrán los datos clínicos provenientes de distintas técnicas médicas. La historia clínica es el lugar por excelencia de la información confiada al profesional y a su correspondiente institución. Conviene recordar, no obstante, que la historia clínica es más que una suma de datos. Es el relato «patográfico» de una persona enferma a quien se puede leer e interpretar como un «texto» que contiene narraciones, es decir, una parte importante de su «biografía».

En cualquier caso, los datos de salud exigen: primero, una condición: el consentimiento de la persona enferma; segundo: una finalidad principal: la asistencia sanitaria; tercero: una garantía: la salud y la intimidad del enfermo; y cuarto: una regla moral: respetar su carácter confidencial y no divulgarlo sin previa autorización.

Por otra parte, la confidencialidad es la versión actual del secreto médico que ha sido durante muchos siglos un deber individual sujeto a la discreción de cada profesional. Hoy día, la obligación de guardar secreto está basada en la necesidad de proteger el derecho a la intimidad y el derecho a la protección de datos de salud, implicando directamente a todos los profesionales que intervienen en el acto clínico. Particular relevancia tiene el secreto 
compartido cuando el profesional responsable de un paciente comparte su información con el equipo de profesionales que atiende a ese mismo paciente y que, por esa razón, deben ser «confidentes necesarios».

El secreto profesional, sin embargo, no es absoluto. Puede ser revelado excepcionalmente cuando estén justificados los siguientes motivos: ${ }^{6}$ por imperativo legal, vinculado al estado de necesidad; por deber profesional, vinculado a la defensa del interés público; y por deber penal o procesal, vinculado al derecho de los otros y referente al deber de no hacerles daño.

\section{FUNDAMENTOS ÉTICOS}

La confianza, la lealtad y fidelidad, la autonomía personal y la dignidad humana son los fundamentos de las obligaciones morales respecto a la intimidad y la confidencialidad.

La confianza es seguridad y esperanza firme que se tiene en alguien (profesional) y se tiene de algo (información) como base de la relación clínica y garantía de acceso a información necesaria en el proceso asistencial. Justifica la confidencialidad.

Lealtad es cumplir lo que exige la fidelidad y ésta consiste en tener fe en otra persona. La principal lealtad y fidelidad del médico, por ejemplo, se debe al paciente y la salud de éste debe anteponerse a cualquier otra conveniencia, lo cual obliga a utilizar la información exclusivamente para curar y cuidar la salud y la vida del enfermo.

La autonomía es la capacidad de facilitar libremente información personal referente a la propia salud, basándose en la confianza de que va a ser confidencial. Si no fuera así tampoco habría intimidad y se produciría una devaluación moral de la persona. Pero la autonomía tiene sus límites en casos de menores o de enfermedad mental, por ejemplo.

Todo ser humano, como «sujeto» y «persona», merece el máximo respeto por razón de su mera condición humana, independientemente de cualquier característica o aptitud particular. Las cosas tienen precio y son medios, las personas tienen dignidad, son fines en sí mismas y no se deben instrumentalizar.? La dignidad humana es el fundamento de la intimidad y la confidencialidad, lo que exige tener en cuenta que: 1) la dignidad concentra el valor intrínseco de cada ser humano y es inseparable del sujeto, es esencial, no accidental; 2) los derechos humanos se derivan de la dignidad en el sentido de que no son concesiones políticas, son los mismos para todosy por ello, nos hacen iguales a todos; y 3) el respeto a la dignidad es el principio matriz de la bioética clínica ${ }^{8}$ del que surgen varios principios éticos básicos ${ }^{9}$ donde se insertan los deberes sobre la intimidad y la confidencialidad:

- En todas tus decisiones y actuaciones debes tomar en consideración a todas las personas que puedan verse afectadas o, con otras palabras, «da a cada uno lo suyo» (Ulpiano) y «trata a los demás como quieres que te traten a ti» (regla de oro).

- Todos estamos obligados a respetar la dignidad de los otros como seres humanos o, en otros términos, es necesario adoptar una actitud personal mediante la que reconocemos, afirmamos y nos interesamos por los demás, basándonos en que tienen valor intrínseco, son valiosos en sí mismos y por ello, tienen dignidad.

- Asimismo, estamos obligados a respetar a los demás como personas o, dicho de otro modo, la dignidad de los seres humanos exige adoptar actitudes positivas que consisten en respetar como persona a cualquiera que resulte afectado por nuestro comportamiento. De ahí se derivan exigencias morales concretas como la de respetar su intimidad y guardar confidencialmente la información al respecto.

\section{INTIMIDAD, CONFIDENCIALIDAD Y RIESGOS DE DESHUMANIZACIÓN}

El ser humano es una realidad viviente y compleja de múltiples facetas. Es una realidad poliédrica. Pero, por encima de todo, se experimenta a sí mismo y se manifiesta al exterior como unidad psicosomática. «El cuerpo es la persona», decía el filósofo y teólogo Dietrich Bonhoeffer, ${ }^{10}$ poniendo así de relieve, por un lado, que el cuerpo es mucho más que un ordenado complejo de células, el cuerpo es persona, y por otro lado, la persona no es sólo espíritu, interioridad o alma, la persona es cuerpo. Dicho con otros términos, la persona es una y única realidad biopsicosocial. ." La intimidad 
y la confidencialidad son dos de sus expresiones o facetas más distintivas.

En el ámbito sanitario puede olvidarse el significado ético de la intimidad y la confidencialidad, no por deformación de los profesionales, sino por la marcha habitual de las cosas que lleva a descuidar esos aspectos. Por eso es necesario ser autocríticosy vigilar la presencia diaria de ciertos riesgos que deshumanizan la atención sanitaria:127) la reducción del enfermo a sus datos personales, a siglas y números, puede ignorar la realidad personal unitaria de cada enfermo; 2) la curiosidad o inclinación a enterarse de las cosas ajenas puede conducir a entremeterse sin motivos en la vida privada de terceros; 3) la indiscreción o falta de reserva, prudencia y sensatez en lo que se habla y se dice acerca de otras personas puede hacer un grave daño a su intimidad; y 4) el desarrollo innegable de la telemedicina presenta nuevas formas y nuevos retos al valor ético de la intimidad y la confidencialidad. ${ }^{13}$

\section{INTIMIDAD, CONFIDENCIALIDAD Y PANDEMIA}

A lo largo de este tiempo difícil ha quedado demostrado que la vulnerabilidad e interdependencia de los seres humanos son hechos incontestables. En tal situación, la intimidad y la confidencialidad, como valores éticos y derechos fundamentales, deberían ser objeto del máximo respeto. Dicho lo anterior, las normas reguladoras de esos valores y derechos contemplan excepciones que, como regla general, se materializan en forma de salvaguardas para permitir legítimamente los tratamientos de datos personales en situaciones de emergencia sanitaria de alcance global. ${ }^{14}$

\section{UN DECÁLOGO PARA LA PROTECCIÓN DE DATOS PERSONALES: ${ }^{15}$}

1. Trata los datos de los pacientes como querrías que tratasen los tuyos.

2. ¿Estás seguro de que tienes que acceder a esa historia clínica? Piénsalo. Sólo debes acceder si es necesario para los fines de tu trabajo.

3. El sistema registra tus accesos a la documentación clínica. Se sabe en qué momento y a qué información has accedido. Los accesos son auditados posteriormente.
4. Evita informar a terceros sobre la salud de tus pacientes, salvo que éstos lo hayan consentido o tengas una justificación lícita.

5. Cuando salgas del despacho, asegúrate de cerrar la sesión abierta en tu ordenador. No facilites a nadie tu clave y contraseña; si necesitas un acceso urgente, contacta con el departamento de informática.

6. No envíes información con datos de salud por correo electrónico o por cualquier red pública o inalámbrica de comunicación electrónica; si fuera imprescindible, no olvides cifrar los datos.

7. No tires documentos con datos personales a la papelera; destrúyelos tú mismo o sigue el procedimiento establecido en tu centro.

8. Cuando termines de pasar consulta, cierra con llave los armarios o archivadores que contengan información clínica.

9. No dejes las historias clínicas a la vista sin supervisión.

10. No crees por tu propia cuenta ficheros con datos personales de pacientes; consulta siempre antes con el departamento de informática.

Recuérdese lo que afirmaba Séneca: Si quieres guardar un secreto, guárdalo tú mismo.

\section{Biblografía}

1. Fundación de Ciencias de la Salud. Cuías de ética en la práctica médica. Intimidad, confidencialidad y secreto. Madrid; 2005. (Acceso 2018-02-05). Disponible en: https:// uww.cgcom.es/sites/default/files/guia_confidencialidad.pdf.

2. Reglamento 2016/679 del Parlamento Europeo y del Consejo, de 27 de abril de 2016, relativo a la protección de las personas físicas en lo que respecta al tratamiento de datos personales y a la libre circulación de estos datos y por el que se deroga la Directiva 95/46/CE. (Acceso 25 Febrero 2018). Disponible en: https://www.boe.es/doue/2016/119/ L00001-00088.pdf.

3. Morais Y. Secret médical. En: Hottois JNG. Missa (dirs.). Nouvelle encyclopédie de bioéthique. Éditions De Boeck Université: Bruxelles; 2001, p. 726.

4. Laín EP. La enfermedad humana. En: Albarracín A (ed). Historia de la enfermedad. Madrid; 1986, pp. 9-17.

5. Orden SSI/87/2017, de 19 de enero. Protocolo mediante el que se determinan pautas básicas destinadas a asegurar y proteger el derecho a la intimidad del paciente por los alumnos y residentes en Ciencias de la Salud, Art. 4, 4.7. (Acceso 2018-02-25). Disponible en: https://www.boe.es/ boe/dias/2017/02/06/pdfs/BOE-A-2017-1200.pdf. 
6. Consejo General de Colegios Oficiales de Médicos. Código de Deontología Médica. Madrid; 2011: Art. 30. (Acceso 201803-01). Disponible en: http://www.cgcom.es/sites/default/ files/codigo_deontologia_medica_0.pdf.

7. Kant I. Fundamentación de la metafísica de las costumbres. Madrid: Espasa Calpe-Austral; 1990, pp. 100-119.

8. Andorno R. Bioética y dignidad de la persona. Tecnos: Madrid; 2012, pp. 35-38.

9. Von Kutschera F. Fundamentos de ética. Madrid: Cátedra; 1982, pp. 282-294.

10. Bonhoeffer D. Editorial Trotta Ética. Madrid; 2000.

11. Engel $\mathrm{GL}$. The clinical application of the biopsycosocial model. Am J Psychiatry. 1980; 137: 535-544.

12. Fernández RE. Intimidad y confidencialidad en la relación clínica. Persona y Derecho. 2013; 69 (2): 70-73. (Acceso 2018-03-01). Disponible en: https://www.unav. edu/publicaciones/revistas/index.php/persona-y-derecho/ article/viewFile/1572/1443.

13. Asociación Médica Mundial. Declaración sobre la ética de la telemedicina (Acceso 2019-02-17). Disponible en: https:// www.wma.net/es/policies-post/declaracion-de-la-ammsobre-la-etica-de-la-telemedicina/.

14. Comisión Asesora de Bioética del Principado de Asturias. Reflexiones éticas para una situación de emergencia Covid-19. Oviedo, 2020 (Acceso 202006-24). Disponible en: https://www.astursalud.es/en/ noticias/-/noticias/informes-de-la-comision-asesorade-bioetica.

15. Agencia Española de Protección de Datos. Madrid, 2017 (Acceso 2018-03-02). Disponible en: http://www.agpd.es/ portalwebAGPD/canaldocumentacion/publicaciones/ common/Guias/2017/DecalogoPersonalSanitario.pdf. 